\title{
ON HAUSDORFF DIMENSION OF RECURRENT NET FRACTALS
}

\author{
SERGIO STELLA
}

(Communicated by Kenneth R. Meyer)

\begin{abstract}
We estimate the Hausdorff dimension of recurrent net fractals showing that it coincides with the box-counting dimension. This is done for geometric constructions in a complete metric space, generalizing well-known theorems about self-similar sets. In particular, it follows that what is really essential in the dimension estimates of self-similar sets are their metric features and not the dynamical ones.
\end{abstract}

\section{INTRODUCTION AND DEFINITIONS}

A great variety of fractals can be obtained through a process that is a direct generalization of the method of construction of the Cantor ternary set, see Mandelbrot [11]. By this method, the fractal set is viewed as the kernel of a geometric scheme defined recursively. Usually, the sets involved in the construction are compact subsets of a complete metric space $\Omega$, and the set obtained through this process is a compact, perfect subset of $\Omega$. In many cases it is totally disconnected and this is always the case when the compact sets forming the scheme are disjoint.

Most of studied cases of such constructions are those in which the geometric scheme is defined dynamically by a finite set of contractions, see Hutchinson [9]. In this framework the fractal is viewed as the attractor of a special dynamical system called Iterated Function System (IFS, in the sequel). For an account of this approach, see Barnsley and Demko [2] and, for consequential applications to fractal interpolation, see Barnsley [1].

Successively, this setting was expanded in order to include in it the recurrent sets, by considering them as attractors of generalized orbits conceived as random walks. See Barnsley, Elton, and Hardin [3] and Bedford [4].

A fractal set, generally, has a complicated structure at every scale and in some sense this geometrical complexity is represented by the dimension of the fractal.

Although there is no general agreement about the definition of the dimension of a fractal, the Hausdorff dimension and the box dimension can be considered the most widely used. The former is preferred because of its theoretical meaning, while the latter is used more often in concrete applications because it is easier to estimate.

Received by the editors June 13, 1990 and, in revised form March 4, 1991.

1980 Mathematics Subject Classification (1985 Revision). Primary 28A75.

Key words and phrases. Dimension, fractal, self-similar set, recurrent net fractal. 
In the dynamical setting, the dimension calculation has been performed by several authors inspired by Hutchinson's elegant treatment [9]. For an account of the technique involved, generalizations and improvements, the papers of Barnsley, Elton, and Hardin [3], Bedford [5], and Mauldin and Williams [12], can be consulted.

These geometric constructions, which are defined dynamically by a finite set of maps, all share the characteristic properties of a net. According to Falconer [7], we term net fractals those sets that are defined, not necessarily in a dynamical way, as intersections of sets belonging to a net.

In this note we consider recurrent net fractals whose scaling properties are approximated from below and from above in a simple and natural way. We show by elementary techniques, that for this type of fractals the Hausdorff dimension and the box dimension are equal estimating them from below and from above. Moreover, we are able to obtain well-known dimensions estimates in the dynamical setting, as a direct consequence of our geometric approach showing that what is really essential in the dimension calculation are the metric features of the fractal, not the dynamical. We have preferred to consider the setting in a general complete metric space $\Omega$, because the achievements obtained are immediately applicable to the hyperspace of $\Omega$, that is, to the space where the fractals "live." Moreover, we can achieve the results about the nonrecurrent case as a straightforward corollary.

We define a geometric scheme in $(\Omega, d)$ with the help of a symbolic space.

Fixed, once at all a positive integer $N$ we denote by $\Sigma^{N}$ the set of all infinite sequences, called indexes, $\mathbf{i}=\left(i_{1}, i_{2}, \ldots\right)$ with $i_{j} \in\{1,2, \ldots, N\} \forall j$. We put $i_{1}, \ldots, i_{n}=\mathbf{i} \mid n$ and say that $\mathbf{i} \mid n$ is a curtailed index.

Let $M$ be a $N \times N$ matrix having as entries only the two numbers 0 and 1. Such a matrix is termed transition matrix. A transition matrix is called irreducible if there exists a positive integer $k$ such that $M^{k}>0$, that is, if all the entries of the matrix $M^{k}$ are positive.

We will suppose throughout the paper that the matrix $M$ is irreducible.

An element $\mathbf{i}$ of $\Sigma^{N}$ is said to be $M$-admissible if $M_{i_{j}, i_{j+1}}=1$ holds for all $j \in \mathbf{N}$. An analogous definition holds for the curtailed indexes. We denote by $\Sigma_{M}^{N}$ the set of all $M$-admissible indexes of $\Sigma^{N}$.

In what follows, a property satisfied by a geometrical construction involving the space $\Sigma^{N}$ is often shared by an analogous construction involving the space $\Sigma_{M}^{N}$. When this happens, we use $\Sigma$ to denote both the symbolic spaces $\Sigma^{N}$ and $\Sigma_{M}^{N}$.

Let $(\Omega, d)$ a complete metric space. A geometric scheme is a family $\left\{A_{\mathbf{i} \mid n}\right\}_{\mathbf{i} \in \Sigma}$ indexed in the symbolic space $\Sigma$, where for any curtailed index $\mathbf{i} \mid n$, the set $A_{\mathbf{i} \mid n}$ is a not empty compact subset of $\Omega$, such that $\overline{\operatorname{int}\left(A_{\mathbf{i} \mid n}\right)}=A_{\mathbf{i} \mid n}$, satisfying the following properties:

$$
\begin{array}{rll}
\text { (i) } \operatorname{int}\left(A_{\mathbf{i} \mid n, k}\right) \cap \operatorname{int}\left(A_{\mathbf{i} \mid n, j}\right)=\varnothing & \forall k \neq j, \forall i \in \Sigma^{N}, \forall n . \\
\text { (ii) } A_{i_{1}, \ldots, i_{n}, i_{n+1} \subseteq A_{i_{1}}, \ldots, i_{n}} & \forall i \in \Sigma^{N} \forall n . \\
\text { (iii) } \lim _{n \rightarrow \infty} d\left(A_{i_{1}}, \ldots, i_{n}\right)=0 & \forall i \in \Sigma^{N} .
\end{array}
$$

From these conditions, one immediately can see that the family $\left\{A_{\mathbf{i} \mid n}\right\}_{\mathbf{i}}$ is indeed a net. The kernel $\mathscr{N}$ of a geometric scheme $\left\{A_{\mathbf{i} \mid n}\right\}_{\mathbf{i}}$ is the set obtained 
in the following way:

$$
\mathscr{N}=\bigcap_{n=1}^{\infty} \bigcup_{\mathbf{i} \mid n} A_{\mathbf{i} \mid n}
$$

Following Falconer [6] we will say that $\mathscr{N}$ is a net fractal. In particular when the symbolic space is $\Sigma^{N}$ we call the family $\left\{A_{\mathbf{i} \mid n}\right\}_{\mathbf{i}}$ a Cantor scheme and say that $\mathscr{N}$ is a net fractal of Cantor type, while when $\Sigma=\Sigma_{M}^{N}$ we call $\left\{A_{\mathbf{i} \mid n}\right\}_{\mathbf{i}}$ a Markov scheme and say that $\mathcal{N}$ is a net fractal of Markov type or a recurrent net fractal.

From the definition we immediately recognize that $\mathscr{N}$ is a compact perfect set. When $\mathscr{N}$ is of Markov type we need the irreducibility property of the matrix $M$ to guarantee these topological properties. The set $\mathscr{N}$ is not necessarily a totally disconnected set, but if the condition (i) of (1) is verified by the whole sets $A_{\mathbf{i} \mid n}$ then the kernel $\mathscr{N}$ is always totally disconnected.

For the dimension estimates of the kernel of a scheme we need to know which metric relations are satisfied by the sets of the scheme.

We suppose that the geometric scheme $\left\{A_{\mathbf{i} \mid n}\right\}_{\mathbf{i}}$ satisfies the following metric relations.

$$
h_{j}\left|A_{\mathbf{i} \mid n}\right| \leq\left|A_{\mathbf{i} \mid n, j}\right| \leq k_{j}\left|A_{\mathbf{i} \mid n}\right| \quad \forall j=1, \ldots, N,
$$

for all $\mathbf{i}$ in $\Sigma$ and for all $n$, with $0<h_{j} \leq k_{j}<1$, where by $|\cdot|$ we have denoted the diameter.

Further we need a condition which ensures that the family $\left\{A_{\mathbf{i} \mid n}\right\}_{\mathbf{i}}$ provides an efficient covering of the set $\mathscr{N}$. To this end we suppose that there exists a $\lambda>0$ such that

$$
\operatorname{inradius}\left(A_{\mathbf{i} \mid n}\right) \geq \lambda\left|A_{\mathbf{i} \mid n}\right| \quad \forall \mathbf{i}, \forall n .
$$

We now recall some basic facts about the two types of dimension we use in the sequel.

For any set $A \subseteq \Omega$ and $\alpha>0$ the $\alpha$-auxiliary Hausdorff measures are defined as follows. For $\varepsilon>0$ we set

$$
\mathscr{H}_{\varepsilon}^{\alpha}(A)=\inf \left\{\sum_{i=1}^{\infty}\left|U_{i}\right|^{\alpha} \mid A \subseteq \bigcup_{i=1}^{\infty} U_{i}, \quad U_{i} \text { open, }\left|U_{i}\right| \leq \varepsilon\right\} .
$$

The $\alpha$-dimensional Hausdorff measure of $A$ is given by

$$
\mathscr{H}^{\alpha}(A)=\sup _{\varepsilon>0} \mathscr{H}_{\varepsilon}^{\alpha}(A)=\lim _{\varepsilon \rightarrow 0^{+}} \mathscr{H}_{\varepsilon}^{\alpha}(A) .
$$

The Hausdorff measure $\mathscr{H}^{\alpha}$ is a regular outer measure. Standard references on Hausdorff measures and dimensions are the books of Falconer [6] and Rogers [14].

The positive real number $\alpha$ such that $\alpha=\inf \left\{\beta \mid \mathscr{H}^{\beta}(A)=0\right\}$ is said to be the Hausdorff dimension of the set $A$ and is denoted by $\operatorname{dim}_{H}(A)$. It can be shown that $\alpha=\sup \left\{\beta \mid \mathscr{H}^{\beta}(A)=+\infty\right\}$. According to Falconer [6] we say that $A$ is an $\alpha$-set if in the critical value $\alpha$ the $\alpha$-dimensional Hausdorff measure of $A$ is positive and finite. Observe that if $A$ is an $\alpha$-set then $\operatorname{dim}_{H}(A)=\alpha$, but the converse is not generally true. 
The lower and upper box-counting dimensions of a set $A$ are defined as

$$
\underline{\operatorname{dim}}_{B}(A):=\liminf _{\varepsilon \rightarrow 0^{+}} \frac{\log N(\varepsilon)}{|\log \varepsilon|}
$$

and

$$
\overline{\operatorname{dim}}_{B}(A):=\limsup _{\varepsilon \rightarrow 0^{+}} \frac{\log N(\varepsilon)}{|\log (\varepsilon)|},
$$

where $N(\varepsilon)$ is the least number of sets of diameter at most $\varepsilon$ which are needed to cover $A$. By the definitions it easily follows that

$$
\underline{\operatorname{dim}}_{B}(A)=\inf \left\{s \mid \liminf _{\varepsilon>0} \varepsilon^{s} N(\varepsilon)<+\infty\right\}
$$

and

$$
\overline{\operatorname{dim}}_{B}(A)=\inf \left\{s \mid \limsup _{\varepsilon>0} \varepsilon^{s} N(\varepsilon)<+\infty\right\} .
$$

Thinking of $\varepsilon^{s} N(\varepsilon)$ as an $s$-evaluation of a covering of $A$ we immediately get:

$$
\operatorname{dim}_{H}(A) \leq \underline{\operatorname{dim}}_{B}(A) \leq \overline{\operatorname{dim}}_{B}(A) .
$$

If the lower and upper box-counting dimensions of $A$ coincide we say that their common value is the box-dimension of the set $A$ and we will denote it by $\operatorname{dim}_{B}(A)$.

\section{ULTRAMETRIC NET FRACTALS}

Given a geometric scheme $\left\{A_{\mathbf{i} \mid n}\right\}_{\mathbf{i} \in \Sigma}$ we endow its kernel $\mathscr{N}$ with a new metric in a rather natural way.

Putting $d_{i_{1}, \ldots, i_{n}}:=\left|A_{i_{1}, \ldots, i_{n}}\right|$ we get the following scheme of values: $\left\{d_{\mathbf{i} \mid n}\right\}_{\mathbf{i} \in \Sigma}$. This enables us to define a metric $D$ on $\mathscr{N}$.

Let $x, y$ be two points of $\mathcal{N}$. We say that the index $\mathbf{i} \mid n$ is the separation index of $x$ and $y$, whenever $x, y \in A_{\mathbf{i} \mid n}$ and there exist different $j$ and $k$ such that $x \in A_{\mathbf{i} \mid n, j}$ and $y \in A_{\mathbf{i} \mid n, k}$.

We put $D(x, y):=d_{\mathbf{i} \mid n}$ where $\mathbf{i} \mid n$ is the separation level of $x$ and $y$ and $D(x, y)=0$ whenever $x=y$. We have:

Proposition 1.1. $(\mathcal{N}, D)$ is a complete metric space topologically equivalent to the space $(\mathcal{N}, d)$. In particular, $D$ is an ultrametric.

Proof. Suppose that $D(x, y)=0$; then necessarily, there exists an index $\mathbf{i} \in \Sigma$ such that $x, y \in \bigcap_{n=1}^{\infty} A_{\mathbf{i} \mid n}$. But $\bigcap_{n=1}^{\infty} A_{\mathrm{i} \mid n}$ is a singleton, thus $x=y$. The definition of $D$ is symmetric in $x$ and $y$; then $D(x, y)=D(y, x)$.

Let us now check the ultrametric property for $D$. Let $x, y$, and $z$ be distinct points of $\mathscr{N}$, and let $\mathbf{i} \mid n$ be the separation index of $x$ and $y$, while $\mathbf{j} \mid m$ is that one for $y$ and $z$. We can suppose without restriction that $n \leq m$. Thus $A_{j_{1}, \ldots, j_{m}} \subseteq A_{i_{1}, \ldots, i_{n}}$ since $y \in A_{\mathrm{i} \mid n} \cap A_{\mathrm{j} \mid m}$, then $D(y, z)=d_{i_{1}, \ldots, i_{n}, j_{n+1}, \ldots, j_{m}}$ while $D(x, y)=d_{i_{1}}, \ldots, i_{n}$. So the inequality

$$
D(x, z) \leq \max \left\{d_{i_{1}, \ldots, i_{n}}, d_{i_{1}}, \ldots, i_{n}, j_{n+1}, \ldots, j_{m}\right\}=\max \{D(x, y), D(y, z)\}
$$

holds. Thus $(\mathscr{N}, D)$ is an ultrametric space. 
To check the topological equivalence we can limit ourselves to show that the metrics $d$ and $D$ have the same convergent sequences.

In fact, let $\left(a_{n}\right)_{n}$ be a sequence convergent to $a$ with respect to the metric $D$. Having for any $x$ and $y, d(x, y) \leq D(x, y)$ from $d\left(a_{n}, a\right) \leq D\left(a_{n}, a\right)$ we immediately get the convergence of $\left(a_{n}\right)_{n}$ to $a$ with respect to the metric $d$.

Conversely, suppose the $\left(a_{n}\right)_{n}$ converges to $a$ with respect the metric $d$. Then there exists an index $i \in \Sigma$ such that $\{a\}=\bigcap_{n=1}^{\infty} A_{\mathrm{i} \mid n}$. For any $\varepsilon>0$ we can always find an $l$ such that $\forall n \geq l,\left|A_{\mathbf{i} \mid n}\right| \leq \varepsilon$ holds. Hence, $D\left(a, a_{n}\right) \leq$ $\left|A_{\mathbf{i} \mid n}\right| \leq \varepsilon, \forall n \geq l$. From this, the convergence of the sequence $\left(a_{n}\right)_{n}$ to $a$ with respect to the metric $D$, follows.

The above proof remains valid even if we replace the family of values $\left\{d_{\mathbf{i} \mid n}\right\}_{\mathbf{i} \in \Sigma}$ with any other family $\left\{b_{\mathbf{i} \mid n}\right\}_{\mathbf{i} \in \Sigma}$ that shares the following property: for any $\mathbf{i} \in \Sigma,\left(b_{\mathbf{i} \mid n}\right)_{n}$ is a decreasing sequence converging to 0 . A scheme that satisfies this property is called values scheme and the metric associated to it is called net metric.

The net metric $D$ is a suitable choice because it contains the information about the scaling rates of the geometric scheme $\left\{A_{\mathbf{i} \mid n}\right\}_{\mathbf{i}}$ with respect to the original metric $d$.

Although the dimension estimates with respect to an ultrametric are simpler than the estimates with respect to the original metric, we can make no use of this fact because the topological equivalence is not sufficient to guarantee the invariance of the dimension. In order to obtain the dimension invariance we must require that the two metrics involved be metrically equivalent.

However, we can estimate the dimensions with respect to the net metric $D$ leaving for later the task to prove that with respect to the two metrics the dimensions coincide.

We will introduce on $\mathscr{N}$ other two metrics $D_{L}$ and $D_{U}$ carrying the information about the approximation from below and from above of the scaling rates of the geometrical scheme $\left\{A_{\mathbf{i} \mid n}\right\}_{\mathbf{i} \in \Sigma_{M}^{N}}$. Namely, we set

$$
D_{L}(x, y):=h_{i_{1}} \cdots h_{i_{n}} \text { and } D_{U}(x, y):=k_{i_{1}} \cdots k_{i_{n}},
$$

where $h_{j}$ and $k_{j}$ are the lower and upper scaling rates of the scheme $\left\{A_{\mathbf{i} \mid n}\right\}_{\mathbf{i}}$, verifying (2), and $i_{1}, \ldots, i_{n}=\mathbf{i} \mid n$ is the separation index associated with $x$ and $y$.

Observe that Proposition 1.1 remains true if we replace the metric $D$ with $D_{L}$ and $D_{U}$. Moreover, by condition (2), the net metrics satisfy the relations

$$
D_{L}(x, y) \leq D(x, y) \leq D_{U}(x, y) \quad \forall x, y \in \mathscr{N}
$$

while $d(x, y) \leq D(x, y)$ being of the original metric.

Thus the Hausdorff dimensions with respect the three metrics above satisfy respectively the same inequalities (4).

We can use this fact for approximating the Hausdorff dimension of $\mathscr{N}$ with respect to the net metric $D$ whenever the exact scaling rates of the scheme $\left\{A_{\mathbf{i} \mid n}\right\}$ are unknown.

We now consider the case in which the scheme $\left\{A_{\mathbf{i} \mid n}\right\}_{\mathbf{i} \in \Sigma}$ is of Markov type, that is, $\Sigma=\Sigma_{M}^{N}$. We are going to estimate the Hausdorff dimension of the associated recurrent net fractal $\mathscr{N}$. To this end we need some well-known results from the Perron-Frobenius theory about nonnegative matrices. 
Recall that a matrix is said to be nonnegative if all its entries are nonnegative real numbers.

Theorem 2.1 (Perron-Frobenius; Seneta [15]). Let $M$ be any $N \times N$ nonnegative irreducible matrix. Then the following properties hold:

(i) There exist a positive eigenvalue $\lambda(M)$.

(ii) The left and right eigenvectors associated with $\lambda(M)$ are strictly positive.

(iii) $|\lambda|<\lambda(M)$ for any other eigenvalue $\lambda$ of $M$.

(iv) The dimension of the eigenspace associated with $\lambda(M)$ is 1 .

(v) $\min _{i=1, \ldots, N} \sum_{j=1}^{N} M_{i, j} \leq \lambda(M) \leq \max _{i=1, \ldots, N} \sum_{j=1}^{N} M_{i, j}$ and $\min _{j=1, \ldots, N} \sum_{i=1}^{N} M_{i, j} \leq \lambda(M) \leq \max _{j=1, \ldots, N} \sum_{i=1}^{N} M_{i, j}$.

The positive real number $\lambda(M)$ is also called the Perron-Frobenius eigenvalue of the matrix $M$.

Given a Markov scheme $\left\{A_{\mathrm{i} \mid n}\right\}_{\mathrm{i} \in \Sigma_{M}^{N}}$ satisfying the metric relations (2), we associate to its lower and upper scaling rates the matrices

$$
H^{\alpha}=\left(\begin{array}{cccc}
h_{1}^{\alpha} & 0 & \cdots & 0 \\
0 & h_{2}^{\alpha} & \cdots & 0 \\
\vdots & \vdots & \ddots & \vdots \\
0 & 0 & \cdots & h_{N}^{\alpha}
\end{array}\right), \quad K^{\beta}=\left(\begin{array}{cccc}
k_{1}^{\beta} & 0 & \cdots & 0 \\
0 & k_{2}^{\beta} & \cdots & 0 \\
\vdots & \vdots & \ddots & \vdots \\
0 & 0 & \cdots & k_{N}^{\beta}
\end{array}\right) .
$$

The theorem, that we are going to state establishes with respect to the metric $D$ the estimates from below and from above of the Hausdorff dimension of the recurrent net fractal $\mathscr{N}$, which is the kernel of the Markov scheme $\left\{A_{\mathbf{i} \mid n}\right\}_{\mathbf{i} \in \Sigma_{M}^{N}}$.

We denote by $\operatorname{Dim}_{H}(\mathscr{N})$ the Hausdorff dimension of the kernel $\mathscr{N}$ estimated with respect to the net metric $D$.

Theorem 2.2. Let $\mathcal{N}$ be the kernel of the geometric scheme of Markov type $\left\{A_{\mathbf{i} \mid n}\right\}_{\mathbf{i} \in \Sigma_{M}^{N}}$ satisfying the relations (2) and having $M$ as irreducible transition matrix. Then we have

$$
\alpha \leq \operatorname{Dim}_{H}(\mathscr{N}) \leq \beta,
$$

provided that $\alpha$ and $\beta$ satisfy

$$
\lambda\left(M H^{\alpha}\right)=1, \quad \lambda\left(M K^{\beta}\right)=1,
$$

where $H^{\alpha}$ and $K^{\beta}$ are the diagonal matrices (5).

Proof. First we define a variant of the lower and upper net metrics: Let $\left(v_{1}, \ldots, v_{N}\right)$ and $\left(w_{1}, \ldots, w_{N}\right)$ be the left eigenvectors associated with the matrices $M H^{\alpha}$ and $M K^{\beta}$ respectively. Moreover, we can suppose that they are normalized in the sense that $\sum_{j=1}^{N} v_{j}=1$ and $\sum_{j=1}^{N} w_{j}=1$.

Define $D_{L}^{\prime}$ and $D_{U}^{\prime}$ as the net metrics whose values schemes are

$$
\left\{h_{i_{1}} \cdots \cdot h_{i_{n}} \cdot v_{i_{n}}^{1 / \alpha}\right\}_{\mathrm{i} \in \Sigma_{M}^{N}} \text { and }\left\{k_{i_{1}} \cdots \cdot k_{i_{n}} \cdot w_{i_{n}}^{1 / \beta}\right\}_{\mathbf{i} \in \Sigma_{M}^{N}} .
$$

By the metric relations (2) we immediately get

$$
c \cdot D_{L}^{\prime}(x, y) \leq D(x, y) \leq C \cdot D_{U}^{\prime}(x, y) \quad \forall x, y \in \mathscr{N},
$$

with $c$ and $C$ suitable constants. 
Consider now the $\alpha$-evaluation of $\mathscr{N}$ with respect to the net metric $D_{L}^{\prime}$; we have

$$
D_{L}^{\prime}\left(A_{i}\right)^{\alpha}:=h_{i}^{\alpha} v_{i}
$$

and recursively we get

$$
D_{L}^{\prime}\left(A_{i_{1}}, \ldots, i_{n, j}\right)^{\alpha}=D_{L}^{\prime}\left(A_{i_{1}}, \ldots, i_{n}\right)^{\alpha} h_{j}^{\alpha} v_{j} / v_{i_{n}} .
$$

Then we obtain

$$
\begin{aligned}
\sum_{j:(\mathbf{i} \mid n, j) \in M \text {-adm }} D_{L}^{\prime}\left(A_{\mathbf{i} \mid n, j}\right)^{\alpha} & =D_{L}^{\prime}\left(A_{\mathbf{i} \mid n}\right)^{\alpha} \sum_{j:(\mathbf{i} \mid n, j) \in M \text {-adm }} h_{j}^{\alpha} v_{j} / v_{i_{n}} \\
& =D_{L}^{\prime}\left(A_{\mathbf{i} \mid n}\right)^{\alpha} \sum_{j} M_{i_{n}, j} h_{j}^{\alpha} \frac{v_{j}}{v_{i_{n}}} \\
& =D_{L}^{\prime}\left(A_{\mathbf{i} \mid n}\right)^{\alpha} \frac{v_{i_{n}}}{v_{i_{n}}} \lambda\left(M H^{\alpha}\right)=D_{L}^{\prime}\left(A_{\mathbf{i} \mid n}\right)^{\alpha},
\end{aligned}
$$

where $M$-adm denotes the set of the curtailed $M$-admissible indexes of $\sum^{N}$. Thus

$$
\sum_{j:(\mathbf{i} \mid n, j) \in M \text {-adm }} D_{L}^{\prime}\left(A_{\mathbf{i} \mid n, j}\right)^{\alpha}=D_{L}^{\prime}\left(A_{\mathbf{i} \mid n}\right)^{\alpha}
$$

holds.

In the same manner it can be proved that

$$
\sum_{j:(\mathbf{i} \mid n, j) \in M-\mathrm{adm}} D_{U}^{\prime}\left(A_{\mathbf{i} \mid n, j}\right)^{\beta}=D_{U}^{\prime}\left(A_{\mathbf{i} \mid n}\right)^{\beta} .
$$

By the inequalities (6), we have $D\left(A_{\mathbf{i} \mid n}\right)^{\beta} \leq D_{U}^{\prime}\left(A_{\mathbf{i} \mid n}\right)^{\beta}$.

Consequently, the $\beta$-evaluations of $\mathscr{N}$ with respect to the net metric $D$ are bounded from above by the $\beta$-evaluations with respect the net metric $D_{U}^{\prime}$, and, with an inductive use of the equality (8), we get

$$
\begin{aligned}
& \sum_{j:(\mathbf{i} \mid n, j) \in M \text {-adm }} D_{U}^{\prime}\left(A_{\mathbf{i} \mid n, j}\right)^{\beta}=D_{U}^{\prime}\left(A_{\mathbf{i} \mid n}\right)^{\beta}=\sum_{j:(\mathbf{i} \mid n-1, j) \in M \text {-adm }} D_{U}^{\prime}\left(A_{\mathbf{i} \mid n-1, j}\right)^{\beta} \\
& =D_{U}^{\prime}\left(A_{\mathbf{i} \mid n-1}\right)^{\beta}=\cdots=\sum_{j:\left(i_{1}, j\right) \in M \text {-adm }} D_{U}^{\prime}\left(A_{i_{1}, j}\right)^{\beta}<+\infty .
\end{aligned}
$$

We obtain in this way the upper bound for the Hausdorff dimension $\operatorname{Dim}_{H}(\mathscr{N})$ $\leq \beta$. For the estimate from below we first have to check that for any open cover of $\mathscr{N}$, say $\{U\}$, we can find a cover of $\mathscr{N}$ made by sets of the scheme $\left\{A_{\mathrm{i} \mid n}\right\}_{\mathrm{i} \in \Sigma_{M}^{N}}$ whose associated $\alpha$-evaluation is not greater than the $\alpha$-evaluation associated with the cover $\{U\}$. This last property is a consequence of the fact that the complement of 0 in the range of the net metric $D$ is a set of isolated points. Thus, for any $U \in\{U\}$ we can find a curtailed index $\mathbf{i} \mid n$ such that $U \subseteq A_{\mathbf{i} \mid n}$ and $D(U)=D\left(A_{\mathbf{i} \mid n}\right)$. With this choice the corresponding evaluations satisfy the relations

$$
\sum D_{L}^{\prime}\left(A_{\mathbf{i} \mid n}\right)^{\alpha} \leq \sum D\left(A_{\mathbf{i} \mid n}\right)^{\alpha} \leq \sum D(U)^{\alpha} .
$$

As in the preceding step an inductive use of the property (7) enables us to conclude with $\alpha \leq \operatorname{Dim}_{H}(\mathcal{N})$. 


\section{Dimensions estimates}

Larman in [10] introduced a theory of dimension suitable for the generalized Hausdorff measures, that is, for the Hausdorff measures having as auxiliary measures

$$
\mu_{\delta}^{h}(A):=\inf \left\{\sum_{i=1}^{\infty} h\left(\left|U_{i}\right|\right)\left|A \subseteq \bigcup_{i=1}^{\infty} U_{i},\right| U_{i} \mid \leq \delta\right\},
$$

where $h:[0,+\infty[\rightarrow[0,+\infty[$ is an increasing function continuous on the right and such that $h(0)=0$. The function $h$ is called a Hausdorff function. Observe that the Hausdorff measure $\mathscr{H}^{\alpha}$ is a particular case of this definition having as Hausdorff function the following $h(t)=t^{\alpha}$.

Larman called finite-dimensional, any metric space $A$ for which there exists a Hausdorff function $h(t)$ such that $\mu^{h}(A)=0$.

Moreover, he proved [10, Theorem 11], that any finite-dimensional compact metric space $A$ is a $\beta$-space; that is, there exists a triple of real numbers $(M, \delta, \alpha)$ with $\alpha<\frac{1}{2}$ such that for all $r \leq \delta$ at most $M$ disjoint open spheres of radius $\alpha r$ can meet any given open sphere of radius $r$.

Theorem 2.2 enables us to say that he kernel $\mathscr{N}$ of a geometric scheme $\left\{A_{\mathbf{i} \mid n}\right\}$ satisfying the metric relations (2) is finite-dimensional. In fact, the kernel $\mathscr{N}$ of a geometric scheme is a compact metric space and since the original metric $d$ and the net metric $D$ satisfy the relation $d(x, y) \leq D(x, y) \forall x, y \in \mathscr{N}$, we get

$$
\operatorname{dim}_{H}(\mathscr{N}) \leq \operatorname{Dim}_{H}(\mathscr{N}) \leq \beta .
$$

Thus for any $\varepsilon>0$ we have $\mathscr{H}^{\beta+\varepsilon}(\mathscr{N})=0$ and then, being $\mathscr{N}$ compact, it is a $\beta$-space.

The following theorem enables us to extend the validity of the estimates given in Theorem 2.2 and shows that for recurrent net fractals the box-counting dimension coincides with the Hausdorff dimension.

Theorem 3.1. Let $\mathscr{N}$ be a recurrent net fractal, kernel of the geometric scheme $\left\{A_{\mathrm{i} \mid n}\right\}_{\mathrm{i} \in \Sigma_{M}^{N}}$ satisfying the metric relations (2). Then we have

$$
\alpha \leq \operatorname{dim}_{H}(\mathscr{N})=\operatorname{dim}_{B}(\mathscr{N}) \leq \beta,
$$

provided that $\alpha$ and $\beta$ are solutions of

$$
\lambda\left(M H^{\alpha}\right)=1, \quad \lambda\left(M K^{\beta}\right)=1,
$$

where $H^{\alpha}$ and $K^{\beta}$ are the diagonal matrices (5).

Proof. First, we show that the Hausdorff dimension of the kernel $\mathscr{N}$ satisfies the claimed estimates.

Since the original metric $d$ is bounded from above by the upper net metric $D_{U}$, which is equivalent to the net metric $D_{U}^{\prime}$, from Theorem 2.2 we immediately get

$$
\operatorname{dim}_{H}(\mathcal{N}) \leq \operatorname{Dim}_{H}(\mathcal{N}) \leq \beta .
$$

For the estimates from below, we proceed as follows. As remarked above $(\mathcal{N}, d)$ is a compact finite-dimensional metric space, thus a $\beta$-space.

Let $(M, \delta, \alpha)$ be the triple of real numbers associated to it. Define $h:=$ $\min \left\{h_{1}, \ldots, h_{N}\right\}$, since $a<\frac{1}{2}$, we can find a positive integer $k$ such that $\alpha^{k} \leq \lambda h$, where $\lambda$ satisfies relation (3). 
Let $\varepsilon>0$ be such that $\varepsilon \leq \delta$, and let $\{U\}$ be a open cover of the kernel $\mathscr{N}$ such that $|U| \leq \varepsilon$. For any open set $U$ of the covering $\{U\}$, we can argue in the following way.

Let $\rho$ be the diameter of $U$; then for any $i \in \Sigma_{M}^{N}$ we can find $n(i) \in \mathbf{N}$ such that

$$
h \rho \leq D_{i_{1}, \ldots, i_{n(i)}} \leq \rho .
$$

Thus $\lambda h \rho \leq \lambda d_{i_{1}, \ldots, i_{n(i)}} \leq \operatorname{inradius}\left(A_{i_{1}}, \ldots, i_{n(i)}\right)$.

Then $A_{i_{1}}, \ldots, i_{n(i)}$ must contain a ball of radius $\lambda h \rho$. Now, $U$ is contained in a ball of radius $\rho$ and every $A_{i_{1}}, \ldots, i_{n(i)}$ contains at least a ball of radius $\lambda h \rho$ with $\alpha \rho^{k} \leq \lambda h \rho$.

Since $\overline{\mathscr{N}}$ is a $\beta$-space we have that at most $M^{k}$ balls of radius $\alpha^{k} \rho$ can meet any ball of radius $\rho$. To see this, it suffices to note that for $k=1$ it is true, and proceeding recursively on $k$ we can reach the claim.

Thus since at most $M^{k}$ balls of radius $\lambda h \rho$ can meet a ball of radius $\rho$, we deduce that at most $M^{k}$ of the sets $\left\{A_{i_{1}}, \ldots, i_{n(i)}\right\}$ can meet $U$.

Denoting by $M-\operatorname{adm}(U)$ the set of indexes whose corresponding level-sets $\left\{A_{i_{1}}, \ldots, i_{n(i)}\right\}$ intersect $U$, we get

$$
\sum_{U \in\{U\}}\left(\sum_{\mathbf{i} \mid n \in M \text {-adm }(U)}\left|A_{\mathbf{i} \mid n(i)}\right|^{s}\right) \leq \sum_{U \in\{U\}}|U|^{s} \cdot M^{k} .
$$

Thus taking the infimum, we get

$$
\mathscr{M}_{\varepsilon}^{s}(\mathscr{N}) \leq M^{k} \cdot \mathscr{H}_{\varepsilon}^{s}(\mathscr{N})
$$

where we have denoted by $\mathscr{M}_{\varepsilon}^{s}$ the $s$-auxiliary Hausdorff measures with respect the net metric $D$. Recalling that $\mathscr{H}_{\varepsilon}^{s}(\mathscr{N}) \leq \mathscr{M}_{\varepsilon}^{s}(\mathscr{N})$ holds, we conclude that the Hausdorff dimension of the net fractal $\mathscr{N}$, estimated with respect to the original metric $d$, is the same as the Hausdorff dimension estimated with respect to the net metric $D$; thus Theorem 2.2 enables us to reach the claim.

Now we show the equality of the two dimensions. For any $\varepsilon>0$ and for any $i \in \Sigma_{M}^{N}$ we can choose a $n(i)$ as above such that $h \varepsilon \leq\left|A_{i_{1}}, \ldots, i_{n(i)}\right| \leq \varepsilon$, and since $\left|A_{i_{1}}, \ldots, i_{n(i)}\right| \leq k_{i_{1}} \cdots \cdots k_{i_{n(i)}} \cdot$ const, we get

$$
\sum_{\mathrm{i} \mid n(i) \in M \text {-adm }}\left|A_{i_{1}, \ldots, i_{n(i)}}\right|^{s} \leq \sum_{\left(i_{1}, \ldots, i_{n(i)}\right) \in M \text {-adm }} k_{i_{1}}^{s} \cdots \cdot k_{i_{n(i)}}^{s} \cdot \text { const } .
$$

Denoting by $\#(\Sigma)$ the cardinality of the set of indexes $\mathbf{i} \mid n(i)$ involved in the above sum, we have

$$
\#(\Sigma) h^{s} \varepsilon^{s} \leq \sum_{\mathbf{i} \mid n(i)}\left|A_{i_{1}}, \ldots, i_{n(i)}\right|^{s} \leq \sum_{\mathbf{i} \mid n(i)} k_{i_{1}}^{s} \cdots \cdots k_{i_{n(i)}}^{s} \cdot \text { const } .
$$

Choosing $s$ such that $s=\operatorname{dim}_{H}(\mathcal{N})=\operatorname{Dim}_{H}(\mathcal{N})$, by Theorem 2.2 we get

$$
\#(\Sigma) h^{s} \varepsilon^{s} \leq \text { const } ;
$$

then we obtain

$$
\#(\Sigma) \leq \text { const } h^{-s} \varepsilon^{-s} .
$$

Let $N(\varepsilon)$ be the least number of sets of diameter at most $\varepsilon$ that cover $\mathscr{N}$. We have

$$
\varepsilon^{s} N(\varepsilon) \leq \varepsilon^{s} \#(\Sigma) \leq \varepsilon^{s} \text { const } h^{-s} \varepsilon^{-s}=\text { const } h^{-s}<+\infty .
$$


So, necessarily, $\overline{\operatorname{dim}}_{B}(\mathscr{N}) \leq s=\operatorname{dim}_{H}(\mathscr{N})$ holds. Recalling that $\operatorname{dim}_{H}(\mathscr{N}) \leq$ $\underline{\operatorname{dim}}_{B}(\mathcal{N}) \leq \operatorname{dim}_{B}(\mathcal{N})$, we can conclude with $\operatorname{dim}_{H}(\mathcal{N})=\operatorname{dim}_{B}(\mathcal{N})$.

For geometric schemes of Cantor type the property expressed in the theorem can be stated as follows:

Corollary 3.2. Let $\left\{A_{\mathbf{i} \mid n}\right\}_{\mathbf{i}}$ be a geometric scheme of Cantor type verifying the metric relations (2). Then for the net fractal $\mathcal{N}$ kernel of the given scheme, we have

$$
\alpha \leq \operatorname{dim}_{H}(\mathscr{N})=\operatorname{dim}_{B}(\mathscr{N}) \leq \beta,
$$

where $\alpha$ and $\beta$ satisfy

$$
\sum_{j=1}^{N} h_{j}^{\alpha}=1 \quad \text { and } \quad \sum_{j=1}^{N} k_{j}^{\beta}=1 .
$$

Proof. The Cantor scheme $\left\{A_{\mathbf{i} \mid n}\right\}_{\mathbf{i}}$ can be thought of as a Markov scheme whose transition matrix $M$ has all 1's as entries. In this last case, we get

$$
M \cdot H^{\alpha}=\left(\begin{array}{cccc}
h_{1}^{\alpha} & h_{2}^{\alpha} & \cdots & h_{N}^{\alpha} \\
h_{1}^{\alpha} & h_{2}^{\alpha} & \cdots & h_{N}^{\alpha} \\
\vdots & \vdots & \ddots & \vdots \\
h_{1}^{\alpha} & h_{2}^{\alpha} & \cdots & h_{N}^{\alpha}
\end{array}\right), \quad M \cdot K^{\beta}=\left(\begin{array}{cccc}
k_{1}^{\beta} & k_{2}^{\beta} & \cdots & k_{N}^{\beta} \\
k_{1}^{\beta} & k_{2}^{\beta} & \cdots & k_{N}^{\beta} \\
\vdots & \vdots & \ddots & \vdots \\
k_{1}^{\beta} & k_{2}^{\beta} & \cdots & k_{N}^{\beta}
\end{array}\right) .
$$

Thus for the property $(\mathrm{v})$ of Perron-Frobenius theorem the equations $\lambda\left(M H^{\alpha}\right)=$ 1 and $\lambda\left(M K^{\beta}\right)=1$ become the following well-known Moran's formulas $\sum_{j=1}^{N} h_{j}^{\alpha}=1$ and $\sum_{j=1}^{N} k_{j}^{\beta}=1$ (see Moran [13]), while the dimension estimates are provided by Theorem 2.2 .

Often, it happens that a geometric scheme verifies the metric relations (2) in which all the scaling rates are equal. Denoting by $k$ the common value of the scaling rates, we have

Corollary 3.3. Let $\left\{A_{\mathbf{i} \mid n}\right\}_{\mathbf{i} \in \Sigma}$ be a geometric scheme whose scaling rates are all equal to the number $k$. Then if $\left\{A_{\mathbf{i} \mid n}\right\}_{\mathbf{i}}$ is of Markov type with transition matrix $M$, we have as estimates for the kernel $\mathcal{N}$,

$$
\operatorname{dim}_{H}(\mathscr{N})=\operatorname{dim}_{B}(\mathscr{N})=\frac{\log \lambda(M)}{-\log k}
$$

while if $\left\{A_{\mathrm{i} \mid n}\right\}_{\mathrm{i}}$ is of Cantor type we have

$$
\operatorname{dim}_{H}(\mathscr{N})=\operatorname{dim}_{B}(\mathscr{N})=\frac{\log N}{-\log k} .
$$

Proof. To reach the claim through the theorem it is sufficient to note that in our hypothesis we have $\lambda\left(M K^{\beta}\right)=\lambda\left(M \cdot k^{\beta} \cdot \mathrm{Id}\right)=k^{\beta} \lambda(M)$. Thus by the theorem,

$$
\operatorname{dim}_{H}(\mathcal{N})=\operatorname{dim}_{B}(\mathscr{N})=\beta=\frac{\log \lambda(M)}{-\log k}
$$

holds. Now, if $\left\{A_{\mathbf{i} \mid n}\right\}_{\mathbf{i}}$ is of Cantor type the transition matrix $M$ has all 1's as entries and thus by property (v) of the Perron-Frobenius theorem, we get $\lambda(M)=N$. 


\section{NET FRACTALS DYNAMICALLY DEFINED}

The self-similar sets introduced by Hutchinson in [9] and their generalizations, the recurrent sets considered in [5] by Bedford, can be considered the best known fractals.

In this section we will show that self-similar sets and recurrent sets are kernels of geometric schemes respectively of Cantor type and of Markov type. Moreover the estimates of the Hausdorff and box dimensions of these sets are performed by means of Theorem 3.1 and its corollaries, showing in this way that what is actually essential in the dimension estimates is the fact that the sets are net fractals.

In a complete metric space $(\Omega, d)$ consider a finite set of contracting similarity maps $T_{j}: \Omega \rightarrow \Omega$ with ratios $k_{j}<1$.

The set $\left\{T_{1}, \ldots, T_{N}\right\}$ is called Iterated Function System and the following well-known theorem due to Hutchinson states the existence and uniqueness of the self-similar set $K$ associated to $\left\{T_{1}, \ldots, T_{N}\right\}$.

Theorem 4.1 (Hutchinson [9]). In a complete metric space $(\Omega, d)$ consider the Iterated Function System $\left\{T_{1}, \ldots, T_{N}\right\}$ formed by contracting similarity maps $T_{j}: \Omega \rightarrow \Omega$ with ratios $k_{j}<1$. Then there exists a compact subset $K$ such that

$$
K=\bigcup_{j=1}^{N} T_{j}(K) .
$$

Further in [9] it is proved that $K$ is the attractor of the discrete dynamical system whose orbits are of the form $T_{i_{1}} \circ \ldots \circ T_{i_{n}}(x):=T_{i_{1}}, \ldots, i_{n}(x)$ with $x \in \Omega$.

A scheme of sets can be associated to the system $\left\{T_{1}, \ldots, T_{N}\right\}$ in the following way:

$$
\begin{aligned}
K_{i} & :=T_{i}(K), \\
K_{\mathrm{i} \mid n} & :=T_{\mathbf{i} \mid n}(K) .
\end{aligned}
$$

Using methods like those in [9], one can check easily that $K=\bigcap_{n=1}^{\infty} \bigcup_{\mathbf{i} \mid n} K_{\mathbf{i} \mid n}$. Moreover, we have

$$
\lim _{n \rightarrow \infty}\left|K_{\mathbf{i} \mid n}\right|=\lim _{n \rightarrow \infty}\left|T_{\mathbf{i} \mid n}(K)\right|=\lim _{n \rightarrow \infty} k_{i_{1}} \cdots k_{i_{n}} \cdot|K|=0 .
$$

Since $K=\bigcup_{j=1}^{N} T_{j}(K)$, as in [9], it can be shown recursively that $K_{\mathbf{i} \mid n}=$ $\bigcup_{j=1}^{N}\left(K_{\mathbf{i} \mid n, j}\right)$ and thus $\left(K_{\mathbf{i} \mid n, j)} \subseteq K_{\mathbf{i} \mid n}\right.$. Then property (ii) and (iii) of (1) hold.

Without further assumptions on $\left\{T_{1}, \ldots, T_{N}\right\}$, in general, property (i) of (1) is not verified. Accordingly to Hutchinson [9], we say that the IFS $\left\{T_{1}, \ldots, T_{N}\right\}$ satisfies the open set condition if there exist a nonempty open set $O$ such that

(i) $\bigcup_{j=1}^{N} T_{j}(O) \subseteq O$,

(ii) $T_{i}(O) \cap T_{j}(O)=\varnothing, i \neq j$.

Moreover we can suppose $O$ to be bounded, as it happens in most of the applications of the theory.

With this further assumption on the system $\left\{T_{1}, \ldots, T_{N}\right\}$ we can build up a new geometric scheme $\left\{A_{\mathbf{i} \mid n}\right\}_{\mathbf{i}}$ having $K$ as its kernel.

We define it by

$$
A_{i}:=\bar{O}_{i}, \quad A_{i_{1}, \ldots, i_{n}}:=\bar{O}_{i_{1}, \ldots, i_{n}},
$$


where $O_{i}:=T_{i}(O)$ and $O_{i_{1}}, \ldots, i_{n}:=T_{i_{1}, \ldots, i_{n}}(O)$. From the open set condition we, immediately, have $\operatorname{int}\left(A_{\mathbf{i} \mid n}\right) \cap \operatorname{int}\left(A_{\mathbf{i} \mid n}\right)=\varnothing$, whenever $\mathbf{i}|n \neq \mathbf{j}| n$; and as above, one can check that $O_{\mathrm{i} \mid n, j} \subseteq O_{\mathrm{i} \mid n}$ and $\lim _{n \rightarrow \infty}\left|O_{\mathrm{i} \mid n}\right|=0$. Moreover $K_{i_{1}, \ldots, i_{n}} \subseteq \bar{O}_{i_{1}, \ldots, i_{n}} \forall i \forall n$. Then

$$
K=\bigcap_{n=1}^{\infty} \bigcup_{\mathbf{i} \mid n} K_{\mathbf{i} \mid n}=\bigcup_{\mathbf{i}} \bigcap_{n=1}^{\infty} K_{\mathbf{i} \mid n}=\bigcup_{\mathbf{i}} \bigcap_{n=1}^{\infty} \bar{O}_{\mathbf{i} \mid n}=\bigcap_{n=1}^{\infty} \bigcup_{\mathbf{i} \mid n} \bar{O}_{\mathbf{i} \mid n}
$$

since $\bigcap_{n=1}^{\infty} K_{\mathrm{i} \mid n}=\bigcap_{n=1}^{\infty} \bar{O}_{\mathrm{i} \mid n}$. Thus $\left\{A_{\mathrm{i} \mid n}\right\}_{\mathrm{i}}$ is a geometric scheme having $K$ as its kernel. Moreover we have

$$
\left|A_{\mathbf{i} \mid n}\right|=\left|T_{\mathbf{i} \mid n}(\bar{O})\right|=k_{i_{1}} \cdots \cdots k_{i_{n}}|\bar{O}|=k_{i_{n}}\left|T_{i_{1}, \ldots, i_{n-1}}(\bar{O})\right|=k_{i_{n}}\left|A_{\mathbf{i} \mid n-1}\right| .
$$

So the metric relations (2) are verified with $h_{j}=k_{j}$, and the property (3) can be checked using the fact that any similarity map transforms open balls in open balls and, since the set $\left\{T_{1}, \ldots, T_{N}\right\}$ is finite we can always find a suitable $\lambda$ satisfying the property (3).

Thus the self-similar set $K$ is a net fractal of Cantor type and, for any irreducible transition matrix $M$ we can build up geometric schemes of Markov type having as kernels recurrent net fractals.

For the dimension estimates of $K$ we now can apply Theorem 3.1 and its corollaries to obtain well-known results included in Hutchinson [9] and Bedford [5]; see also Falconer [8].

\section{REFERENCES}

1. M. Barnsley, Fractal functions and interpolation, Constr. Approx. 2 (1989), 303-329.

2. M. Barnsley and S. Demko, Iterated function systems and the global construction of fractals, Proc. Roy. Soc. London Ser. A 399 (1985), 243-275.

3. M. Barnsley, J. Elton, and D. Hardin, Recurrent iterated function systems, Constr. Approx. 5 (1989), 3-31.

4. T. Bedford, Crinkly curves, Markov partitions and dimensions, Ph.D. Thesis, Warwick University, Warwick, 1984.

5. __ Dimension and dynamics for fractal recurrent sets, J. London Math. Soc. (2) 33 (1986), 89-100.

6. K. J. Falconer, The geometry of fractal sets, Cambridge Univ. Press, Cambridge, 1985.

7. __ Random fractals, Math. Proc. Cambridge Philos. Soc. 100 (1986), 559-582.

8. _ Dimensions and measures of quasi-self-similar sets, Proc. Amer. Math. Soc. 106 (1989), 543-554.

9. J. E. Hutchinson, Fractals and self-similarity, Indiana Univ. Math. J. 30 (1981), 713-743.

10. D. J. Larman, A new theory of dimension, Proc. London Math. Soc. (3) 17 (1967), 178-192.

11. B. B. Mandelbrot, The fractal geometry of nature, Freemann, New York, 1983.

12. R. D. Mauldin and S. C. Williams, Hausdorff dimension in graph directed constructions, Trans. Amer. Math. Soc. 309 (1988), 811-829.

13. P. A. P. Moran, Additive functions of intervals and Hausdorff measure, Proc. Cambridge Philos. Soc. 42 (1946), 15-23.

14. C. A. Rogers, Hausdorff measures, Cambridge Univ. Press, Cambridge, 1970.

15. E. Seneta, Non-negative matrices and Markov chains, Springer-Verlag, New York, 1981.

Dipartimento di Matematica e Informatica, Universitá degli Studi di Udine, via ZANON, 6. 33100 UdINE, ITALY

Current address: Via Emilia, 96, 33100 Udine, Italy 